\title{
Direct Fabrication of Full-Shell X-ray Optics
}

Mikhail V. Gubarev, Brian Ramsey, W. Scott Smith, Jeffery Kolodziejczak, Jacqueline Roche, Charlie Griffith, William Jones, William Arnold,

Carolyn Atkins. 


\section{X-ray Optics after Chandra}

The challenge is to develop the optical fabrication technology capable of producing $x$-ray optics but with an order of magnitude lighter mirrors and at an affordable price.

The challenge can be approached from different directions:

- Very thin mirrors:

- Replicated optics

- Pore Optics

- Figure Correction

- Not that thin full shells, direct fabrication, Chandra-like, Figure correction can be applied too

Mission-design studies have shown that full-shell optics of few-mm shell thickness can provide for scientifically compelling probe-class missions that satisfy the medium-term needs of $x$-ray astronomy. 


\section{Direct Fabrication}

Technique is pioneered by the Astronomical Observatory of Brera, Italy

\begin{tabular}{|c|c|c|c|c|}
\hline Material & $\begin{array}{c}\text { Density } \\
\left(\mathrm{g} / \mathrm{cm}^{3}\right)\end{array}$ & $\begin{array}{c}\text { CTE } \\
\left(10^{-6} / \mathrm{K}^{-1}\right)\end{array}$ & $\begin{array}{c}\text { Ylastic Modulus } \\
\mathrm{GPa}\end{array}$ \\
\hline Fused Silica & 2.2 & 0.5 & 72 & $48^{*}$ \\
\hline Beryllium & 1.8 & 12 & 318 & 240 \\
\hline Al (6061) & 2.7 & 24 & 69 & 276 \\
\hline AlSi & 2.8 & 17 & 90 & 210 \\
\hline AlSi+SiC(30\% by vol) & 2.8 & 14.6 & 120 & \\
\hline
\end{tabular}

Ideally, the mirror shell has low density, low coefficient of expansion (CTE), high modulus of elasticity and high yield strength. It should also be a material that is not too difficult to figure and polish.

- $\mathrm{Be}+\mathrm{NiP}$ (CATS-ISS telescope)

- $\mathrm{Al}+\mathrm{NiP}$

- $\mathrm{AlSi}+\mathrm{NiP}$

Mechanical Properties of Potential Mirror Substrate Materials

*Maximal achievable value. The 'working' value is typically much less and depends on the surface/subsurface condition.

Challenges:

- CTE

The $10{ }^{\circ} \mathrm{C}$ delta corresponds to a stress of $\sim 2 \mathrm{ksi}$ in the nickel coating. So, any

- Electroless vs. electrolithic inherent stress (in the electroformed NiP) should be much less than that OR such that it offsets the CTE mismatch stress, giving even lower stress in the room temperature article.

Have to control the thickness of the NiP deposit on both sides of the mirror shell 


\section{Direct Fabrication}

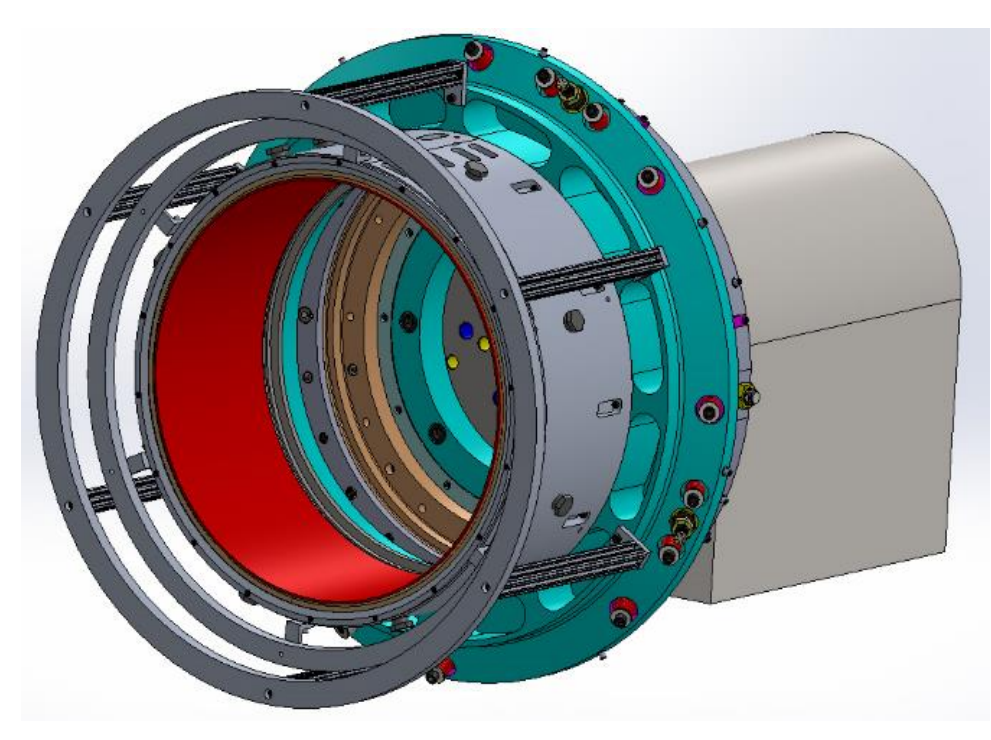

Backing support system installed on the precision lathe for diamond turning. The precision stage (blue) permit alignment of the mirror shell (red) with the lathe.

Thin-shell backing support system. A thin layer of backing material (not shown) acts as interface between the mirror shell (red) and the stiff outer support clamshell (gray). The support rings and the gaskets shown contain the backing material.

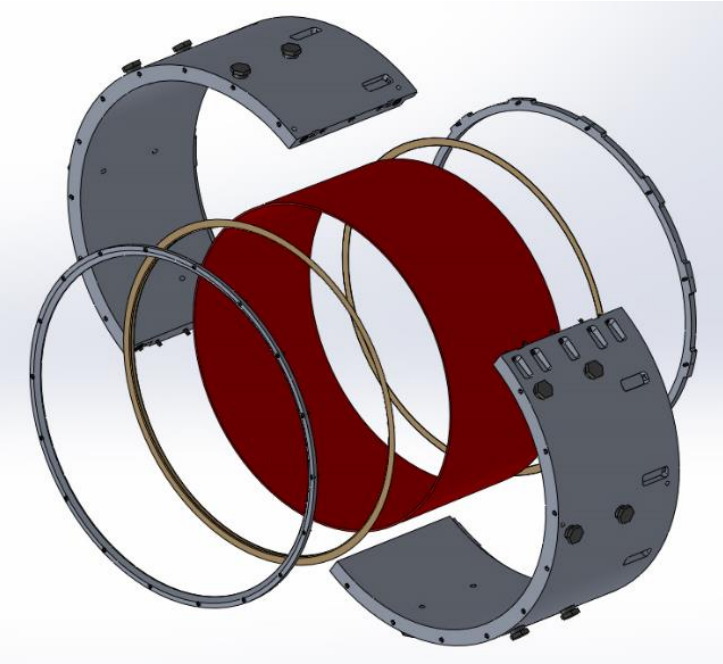

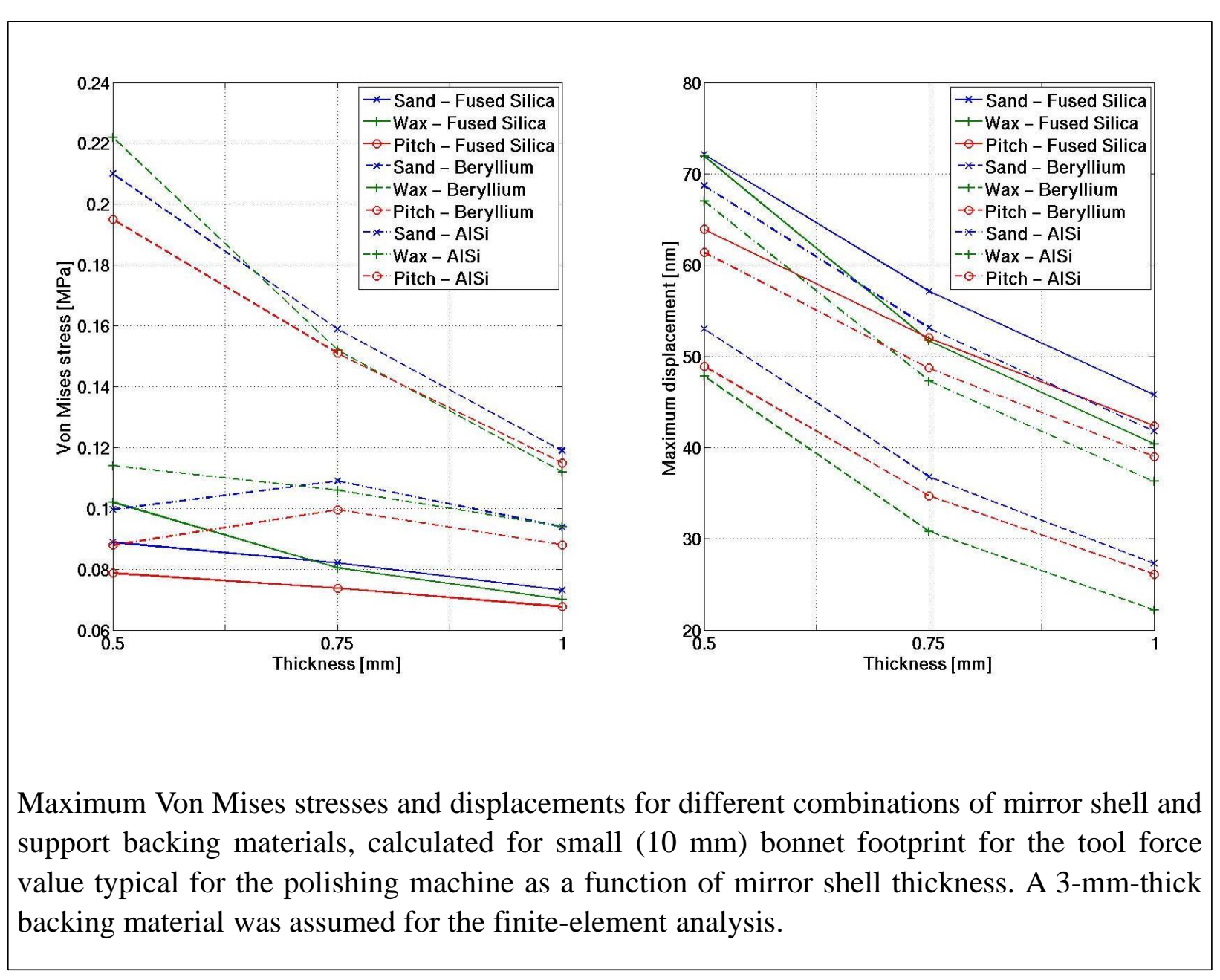




\section{Zeeko machine}

- The machine utilizes a "bonnet" technique in which an inflated rubber hemispherical diaphragm supports the polishing medium.

- there are different "bonnet" sizes (20 $\mathrm{mm}, 40 \mathrm{~mm}$ and $80 \mathrm{~mm}$ radii of curvature)

- This computer-controlled deterministic polishing processes leads to a high convergence rate.

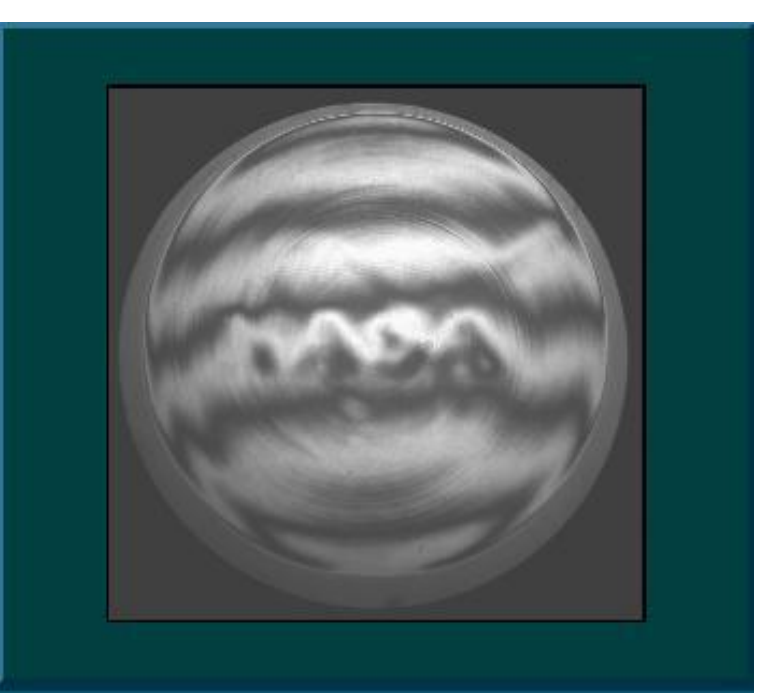

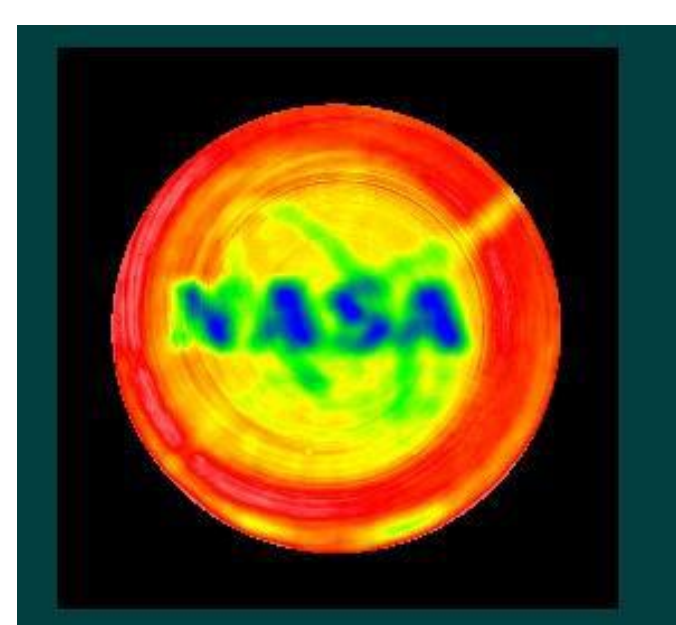

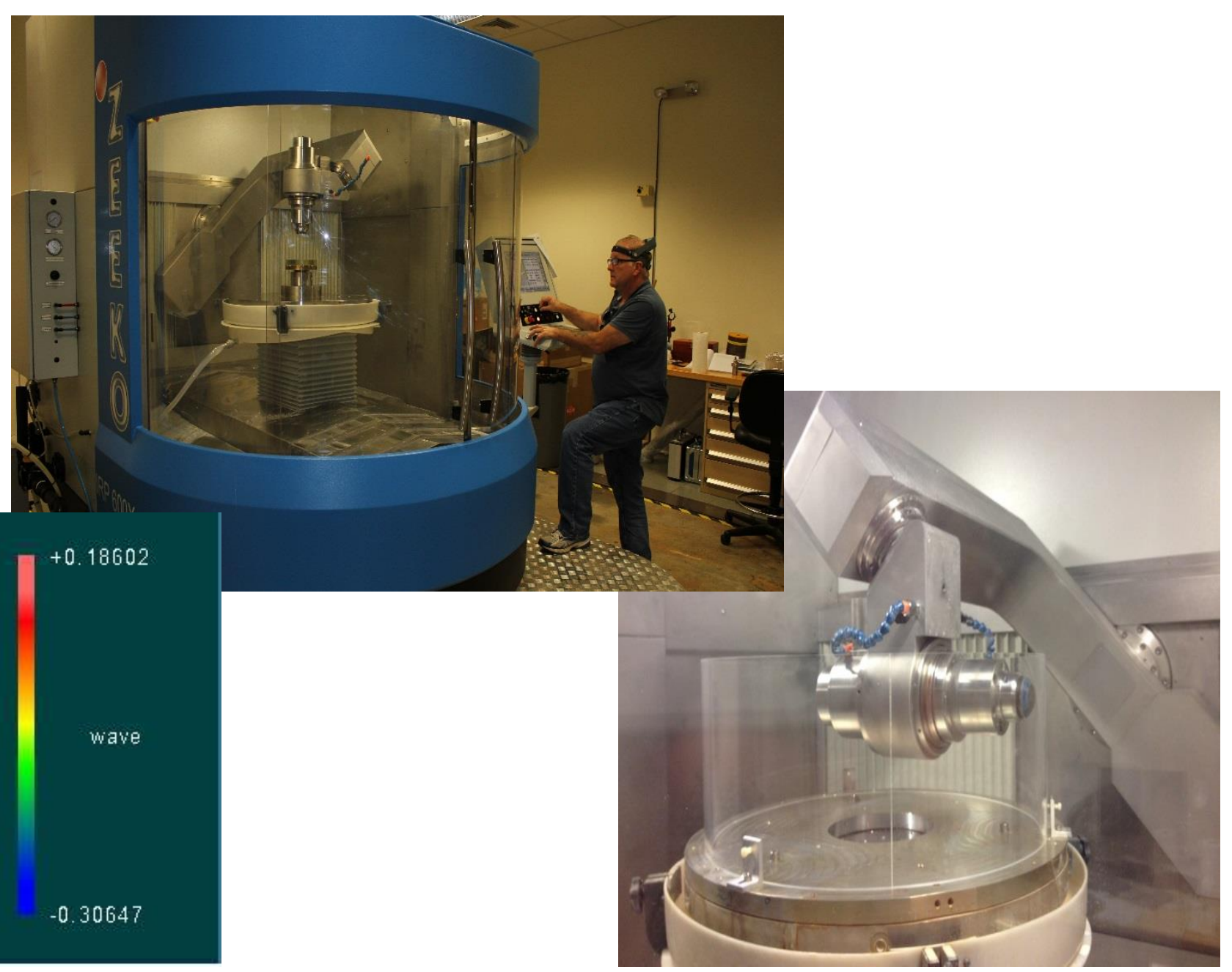




\section{Metrology for Direct Fabrication}

- Vertical Long trace profilometer;

- Skip test;

- Deflectometer for possible in situ measurements

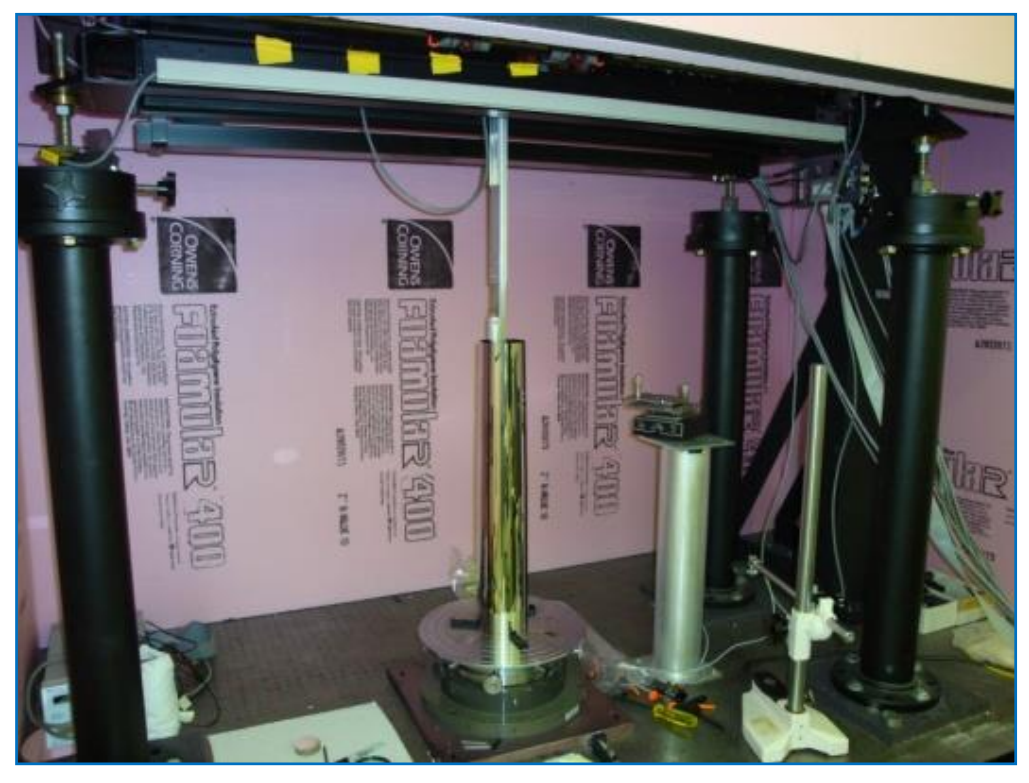

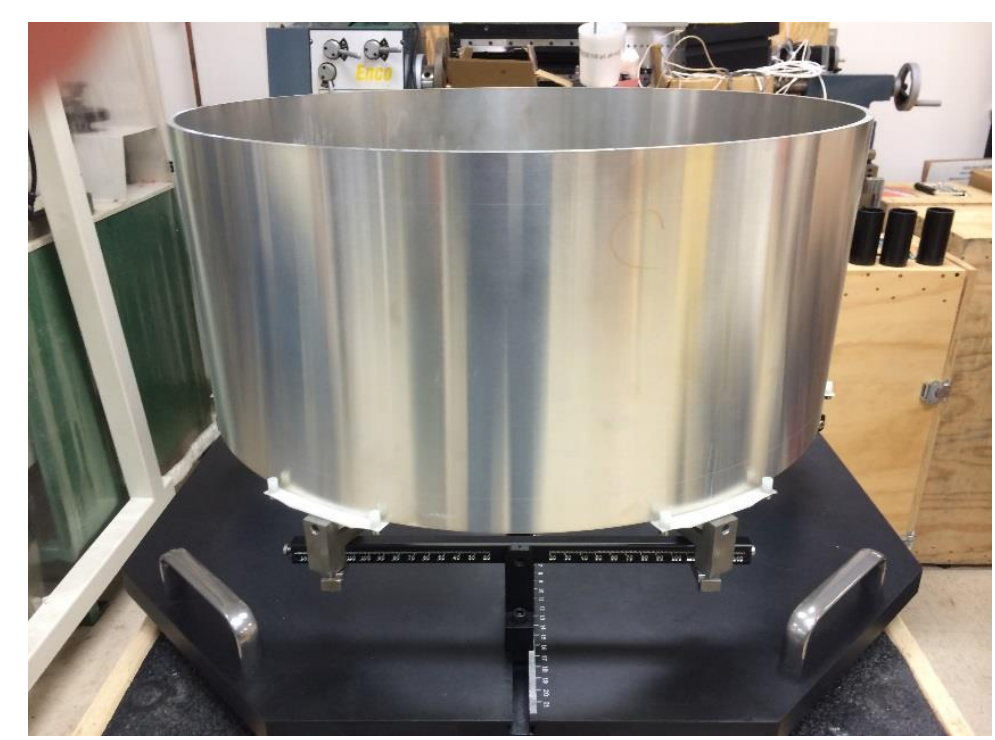

Whiffle tree station with an aluminum shell supported at 12 points.

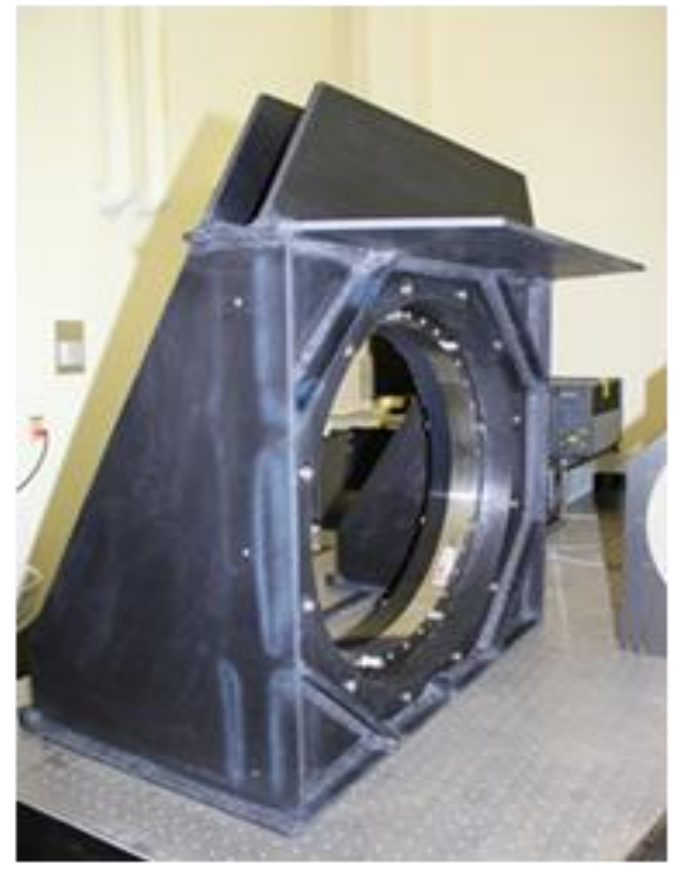

A metrology fixture to support the mirror shell during the skip test. 


\section{CNC Polishing Wear Pattern Characterization}

Currently, we are in the process of characterizing the Zeeko wear function for use in preparing for cylindrical shell polishing runs. Main Objectives:

Determine dependence on machine setup parameters.

Depth and width vs. feed rate

Depth and width vs. bonnet pressure

Depth and width vs. spindle rotation rate

Depth and width vs. tool offset

Depth and width vs. precess and phi angles

Determine dependence on bonnet/cover characteristics.

Determine dependence on slurry characteristics.

Establish a baseline pressure, spindle rotation rate, tool offset

based on simulations

Then perform more finely sampled wear function vs. feed rate at

baseline and $+/$ - small variations to get the derivatives vs. pressure, spindle rotation rate, tool offset.

Determine limiting factors for repeatability

Repeat a baseline test regularly and track changes to bound

variability 


\section{CNC Polishing Wear Pattern Simulation}

Example of Output from Model Run

Parametric wear pattern simulation enables a more efficient method of exploring the polishing parameter space.

Based on Preston's law:

Wear rate is proportional to

Bonnet pressure distribution

Velocity of bonnet surface

Velocity depends on

Spindle rotation

Head attack angles

Pressure depends on

Internal pressure of bonnet

Bonnet structural and mechanical properties

Static (current)

Dynamic (future)

Model will be validated and adjusted using measured data.

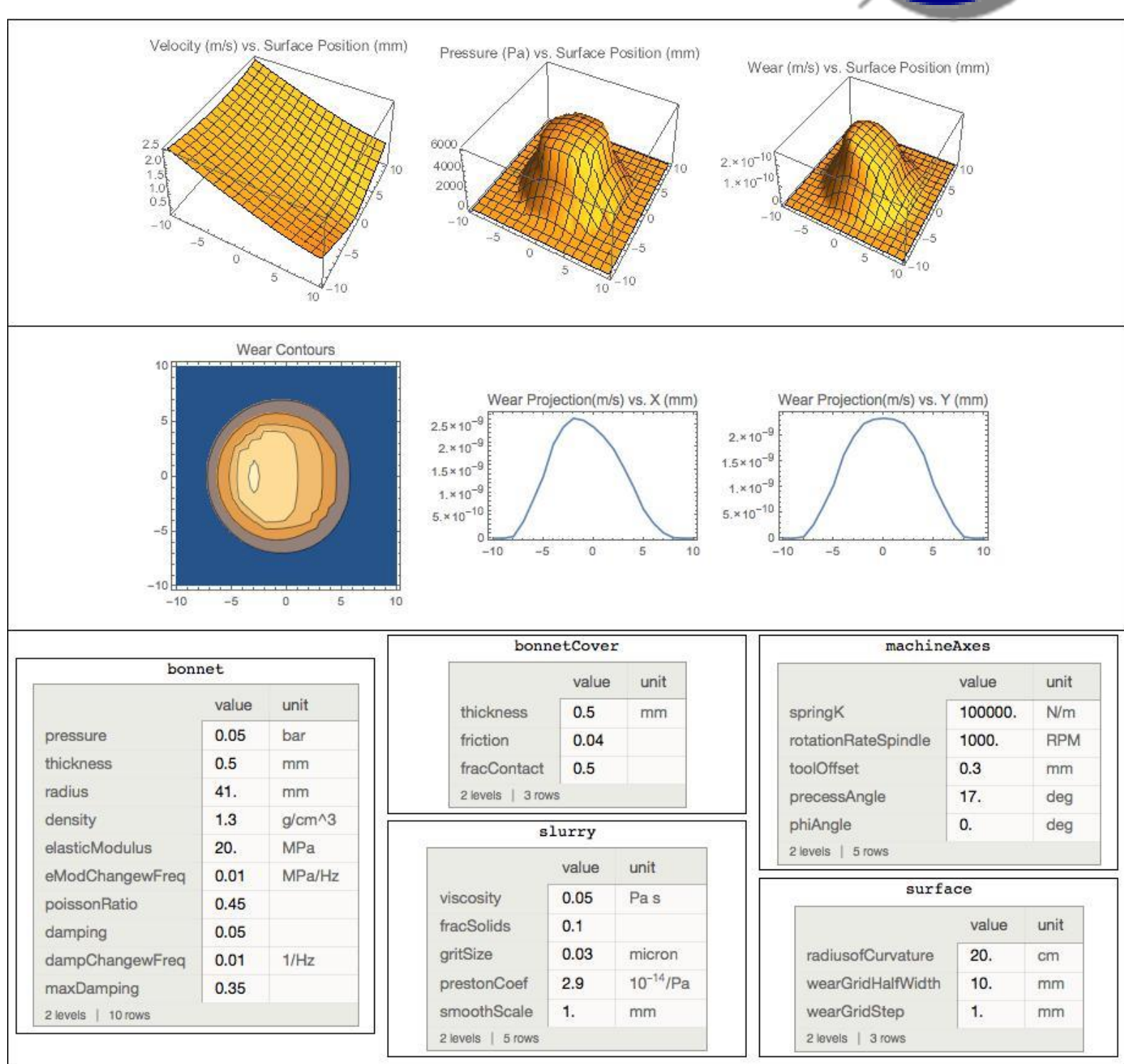




\section{CNC Polishing Wear Pattern Measurement}

Measured wear data from wave front sensor

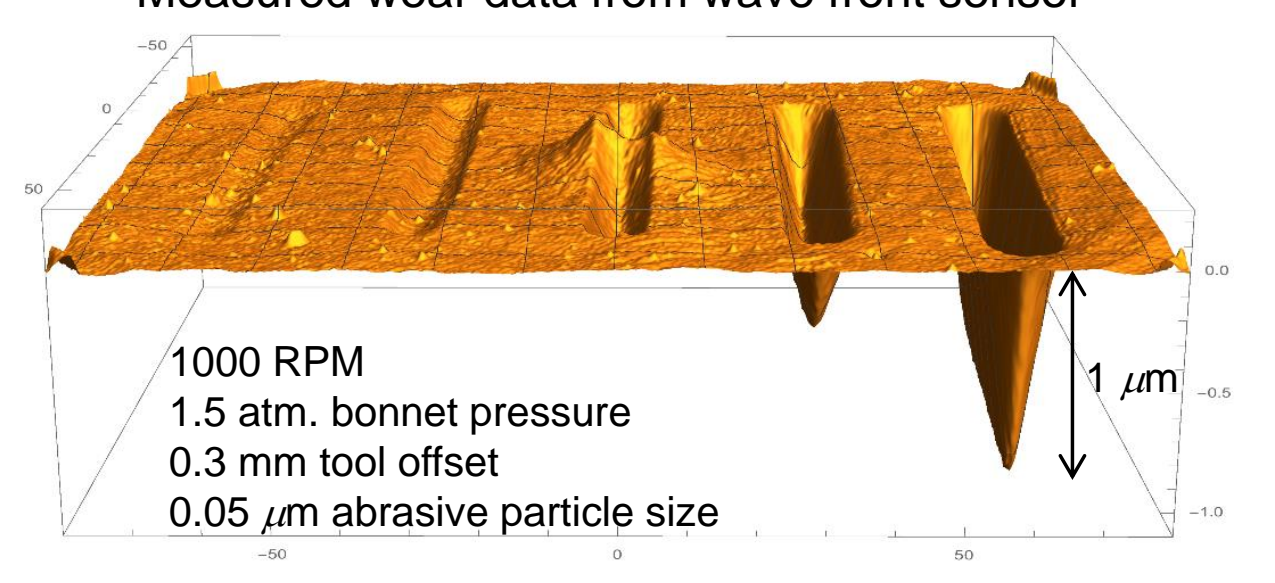

- A priori, simulated profiles are based on a Preston coefficient $=2.9 \times 10^{-14} \mathrm{~Pa}^{-1}$ found in literature. ${ }^{*}$

- Peak projected wear rate $\sim 2.5 \mathrm{~nm} / \mathrm{sec}$.

- Measured wear rate function shows peak $\sim 2.2 \mathrm{~nm} / \mathrm{sec}$

- $\quad$ Agrees well with prior estimate.
- $100 \mathrm{~mm}$ diameter, Ni-P-plated, diamond-turned polishing samples have trenches polished with varying parameters.

- In this case feed rate was varied from 2 to 32 $\mathrm{mm} / \mathrm{min}$

- Wear is measured using Zygo wave front sensor.

- Wear rate function is derived from data.
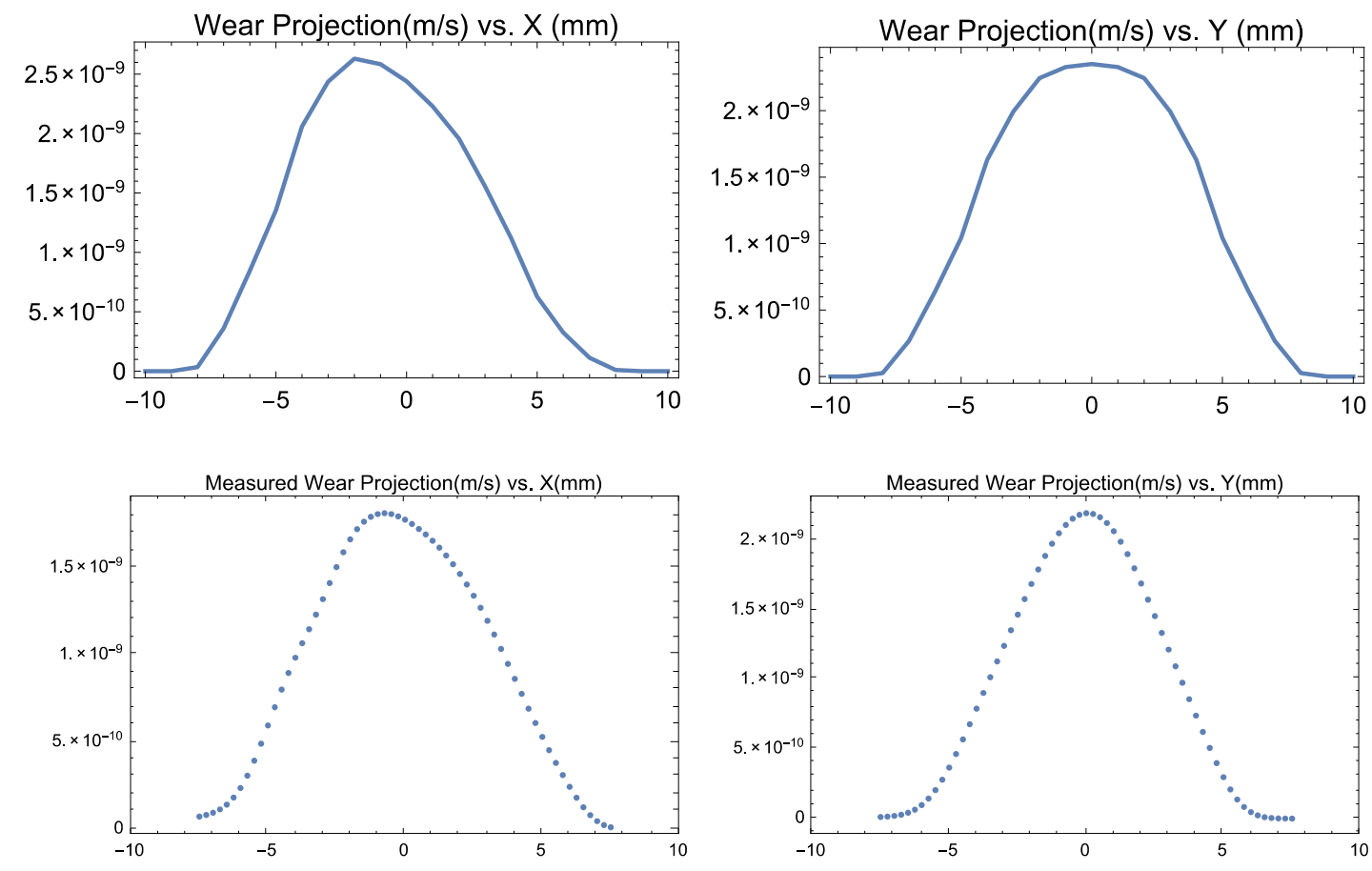


\section{CNC Polishing Wear Pattern Application}

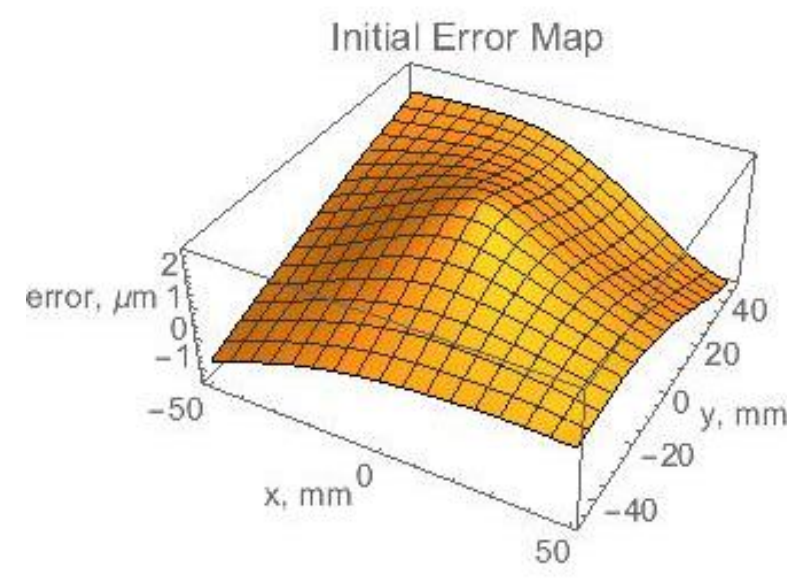

- Initial error map derived from Wyco data.

- RMS slope errors along $\mathrm{x}$-axis are $\mathbf{8}$ arcsec.

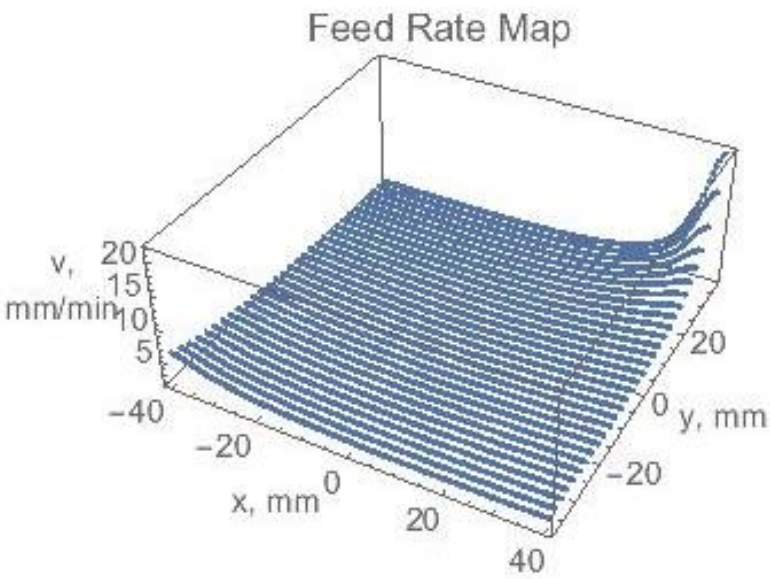

- Derived feed rate map.

- Feed rates range from $1.35-20 \mathrm{~mm} / \mathrm{min}$.

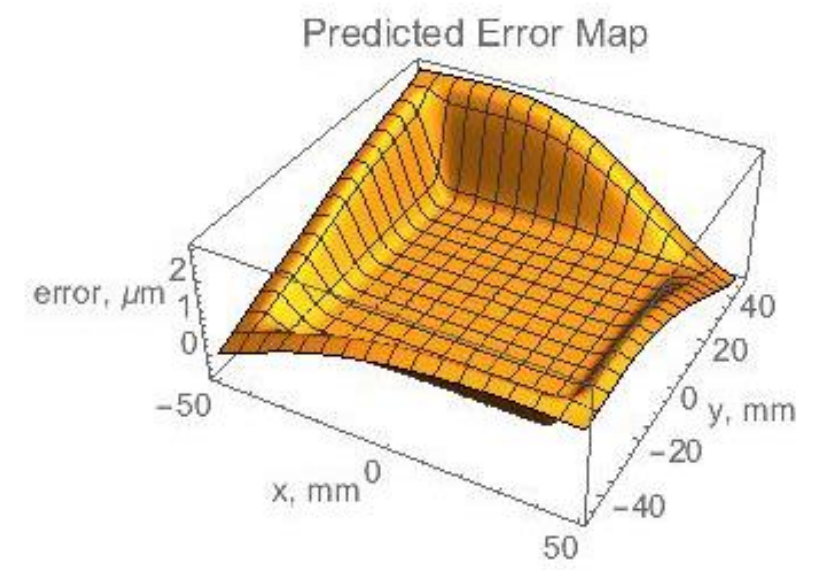

- Predicted result of $1^{\text {st }}$ polishing iteration.

- RMS slope errors along $\mathrm{x}$-axis are predicted to be 1.4 arcsec.

- Measured results await machine repair. 


\section{Conclusions}

- MSFC develops the direct fabrication technology for full shell $x$-ray optics made from metal substrates;

- Support fixtures for diamond-turning, polishing and metrology are designed and currently in production;

- Wear functions were determined using NiP plated flat samples;

- Tool path generation software is developed;

- Electrolithic NiP plated samples are fabricated for the Tool path generation software verification;

- Technology, if developed, will increase competition;

- The technique can be married with the Differential Deposition

- The experiments are pending the machine repair 